\title{
A strategy to identify a ketoreductase that preferentially synthesizes pharmaceutically relevant (S)-alcohols using whole-cell biotransformation
}

Saiful F. Haq ${ }^{1 \dagger}$, Anirudh P. Shanbhag ${ }^{2,7 \dagger}$, Subbulakshmi Karthikeyan ${ }^{1,3}$, Imran Hassan ${ }^{1,8}$, Kannan Thanukrishnan ${ }^{1,4}$, Abhishek Ashok', Sunilkumar Sukumaran', S. Ramaswamy ${ }^{5}$, Nagakumar Bharatham Santanu Datta ${ }^{2}$, Shalaka Samant ${ }^{1}$ and Nainesh Katagihallimath ${ }^{2^{*}(\mathbb{C}}$

\begin{abstract}
Introduction: Chemical industries are constantly in search of an expeditious and environmentally benign method for producing chiral synthons. Ketoreductases have been used as catalysts for enantioselective conversion of desired prochiral ketones to their corresponding alcohol. We chose reported promiscuous ketoreductases belonging to different protein families and expressed them in E. coli to evaluate their ability as whole-cell catalysts for obtaining chiral alcohol intermediates of pharmaceutical importance. Apart from establishing a method to produce high value (S)specific alcohols that have not been evaluated before, we propose an in silico analysis procedure to predict product chirality.

Results: Six enzymes originating from Sulfolobus sulfotaricus, Zygosaccharomyces rouxii, Hansenula polymorpha, Corynebacterium sp. ST-10, Synechococcus sp. PCC 7942 and Bacillus sp. ECU0013 with reported efficient activity for dissimilar substrates are compared here to arrive at an optimal enzyme for the method. Whole-cell catalysis of ketone intermediates for drugs like Aprepitant, Sitagliptin and Dolastatin using E. coli over-expressing these enzymes yielded (S)-specific chiral alcohols. We explain this chiral specificity for the best-performing enzyme, i.e., Z. rouxii ketoreductase using in silico modelling and MD simulations. This rationale was applied to five additional ketones that are used in the synthesis of Crizotinib, MA-20565 (an antifungal agent), Sulopenem, Rivastigmine, Talampanel and Barnidipine and predicted the yield of (S) enantiomers. Experimental evaluation matched the in silico analysis wherein 95\% (S)-specific alcohol with a chemical yield of 23-79\% was obtained through biotransformation. Further, the cofactor re-cycling was optimized by switching the carbon source from glucose to sorbitol that improved the chemical yield to $85-99 \%$.
\end{abstract}

Conclusions: Here, we present a strategy to synthesize pharmaceutically relevant chiral alcohols by ketoreductases using a cofactor balanced whole-cell catalysis scheme that is useful for the industry. Based on the results obtained in these trials, Zygosaccharomyces rouxii ketoreductase was identified as a proficient enzyme to obtain (S)-specific alcohols from their respective ketones. The whole-cell catalyst when combined with nutrient modulation of using sorbitol as a carbon source helped obtain high enantiomeric and chemical yield.

\footnotetext{
*Correspondence: nainesh@bugworksresearch.com

†Saiful F. Haq and Anirudh P. Shanbhag contributed equally to this work

2 Bugworks Research India, Pvt. Ltd, Bengaluru, India

Full list of author information is available at the end of the article
} 
Keywords: Biotransformation, Chiral alcohol synthesis, Short chain dehydrogenase, Medium chain dehydrogenase, Ketoreductase

\section{Background}

Chiral alcohols are essential building blocks in the synthesis of pharmaceutical molecules and fine chemicals [1-3]. Asymmetric reduction of prochiral ketones using enzymatic biotransformation is useful for synthesizing products of enantiomeric purity. In contrast to chemical methods that often do not comply with the principles of green chemistry, biocatalysis offers numerous advantages, such as mild and environmentally benign conditions and remarkable chemo-, regio- and stereoselectivity $[4,5]$. It often facilitates the circumvention of arduous syntheses routes that require multiple protection and deprotection steps [6, 7]. Besides, they can provide an advantage of obtaining up to $100 \%$ theoretical yield with over $99 \%$ enantioselectivity [8]. Biocatalysis for prochiral ketone reduction could either employ a whole-cell system or a purified enzyme preparation [4]. The use of whole-cells offers the advantage of being a simple and low-cost catalyst preparation compared to purified enzymes that are generally expensive due to the need of protein purification, their diminished activity under process conditions, insufficient stability and vulnerability to the substrate and product inhibitions [9]. Enzymes are often more stable within the cellular milieu, as microorganisms can insulate them from harsher environment [10]. Enzymatic reduction of ketones is performed with a stoichiometrically higher proportion of cofactors (NADH or NADPH) as that of the substrate which adds to the cost of synthesis. Cells provide a basal capacity for cofactor regeneration through the reduction of NAD+ and NADP + in central metabolic pathways [11]. The use of whole-cell ketoreductase system also precludes the costly and tedious process of separating the enzymes from the reaction mixture as the products are harvested from media. To identify an efficient enzyme for the process of ketone reduction, we considered two super families of ketoreductases that can convert a broad range of prochiral ketones namely, the medium-chain (MDR) and shortchain (SDR) dehydrogenases/reductases (nineteen enzymes as listed in Table 1 and references therein [12-28].

Table 1 Prospecting ketoreductases from published literature

\begin{tabular}{|c|c|c|c|}
\hline Protein name* & Protein superfamily and family & Amino acids & Organism \\
\hline FabG & NADB Rossmann BKR like & 249 & S. elongatus [12] \\
\hline Scored & NADB Rossmann SDRc & 263 & S. coelicolor [13] \\
\hline Cmafph & NADB Rossmann MDH Like & 254 & C. maris [14] \\
\hline$\angle b R A D H$ & NADB Rossmann $3 \beta-17 \beta$ HSD Like & 257 & L. brevis [15] \\
\hline CpScrll & NADB Rossmann MDH Like & 279 & P. stipites [16] \\
\hline Pscr & NADB Rossmann MDH Like & 282 & P. stipites [17] \\
\hline Hketo & NADB Rossmann MDH Like & 273 & H. polymorpha [18] \\
\hline Cmagsi & NADB Rossmann MDH Like & 283 & C. magnoliae [19] \\
\hline Kacr1 & NADB Rossmann MDH Like & 292 & K. lactis $[20]$ \\
\hline BYueD & NADB Rossmann SPR like SDR & 243 & B. subtilis [21] \\
\hline SSAkr2 & NADB Rossmann AR SDR Like & 323 & S. salmonicolor [22] \\
\hline ZRK & NADB Rossmann AR SDR Like & 338 & Z. rouxii [23] \\
\hline LKadh & MDR FDH Like ADH3 & 347 & L. kefir [24] \\
\hline HVadh2 & MDR FDH Like ADH3 & 349 & H. volacanii [25] \\
\hline HVadh1 & MDR ADH6 & 353 & H. volacanii [25] \\
\hline SsADH & MDR Arabinose DH like & 347 & S. solfataricus [26] \\
\hline SaADHRC3 & MDR Arabinose DH like & 347 & S. solfataricus [27] \\
\hline$P A R$ & MDR Arabinose DH like & 385 & Corynebacteirum sp. [28] \\
\hline SSAkr1 & NADB Rossmann AR SDR Like & 343 & S. salmonicolor [22] \\
\hline
\end{tabular}

Six ketoreductases highlighted in bold were chosen from a set of 19 enzymes based on their presence in different genera and molecular weight, showing varying optomer specific catalysis based on literature

* The nomenclature used for the ketoreductases are from the references denoted in parenthesis 
An often-used technique for engineering enzymes to obtain desired properties of enzyme promiscuity or substrate specificity is DNA shuffling or random mutagenesis [29]. Else, numerous alcohol dehydrogenases [30] or SDRs-Carbonyl reductases [31] are screened to obtain the best enzyme that is useful for a specific substrate. Although these methods have been successfully used they are essentially a 'black-box' approach to obtain an optimal solution. Even minor changes in substrate structure or alignment of amino-acids in the active site drastically alters the specific activity of the enzyme [32]. We demonstrate a rational approach for choosing dehydrogenases that not only circumvents the arduous process of high-throughput screening of shuffled enzymes but aids in making a rational choice of enzymes from different families [33]. To determine the ability of enantioselective conversion, we tested six ketoreductases (highlighted in bold in Table 1) against three high value prochiral pharmaceutical intermediates. We observed that only the ketoreductase Zygosaccharomyces rouxii short chain dehydrogenase/reductase (ZRK) had the required catalytic characteristics that produced the near total $(S)$-enantioselectivity with a robust chemical yield under whole cell bio-transformation. We further investigated the probable reasons for the aforementioned stereo-selective product formation and identified 5 other ketones of pharmaceutical importance that could be catalyzed to produce high value $(S)$-enantiomers. Binding mode of the ketones included in the current study were analyzed by molecular docking and the predictions were further validated by molecular dynamics simulations of ZRK: substrate complex. The in silico predictions have been validated by experimentation.

\section{Results and discussion}

\section{Ketoreductase selection, their over-expression} and solubility in E. coli BL21(DE3)

It is a challenge to design an ideal ketoreductase for maximum chemical yields and enantioselectivity based on the enzyme/substrate structure or sequence alone [33]. Literature studies indicate that short-chain and mediumchain dehydrogenases/reductases (SDRs and MDRs) have high structural similarity despite dissimilar protein sequences. MDRs are 300-400 amino acid long, zinc containing dimeric enzymes. They are well-known reducing agents for non-polar metabolites and act as phase-I detoxification agents $[34,35]$ indicating a high substrate promiscuity, and this is a feature that is useful to convert multiple substrates. SDRs are also dehydrogenases that are 200-300 amino acids long and exist as monomers and have a different mechanism of catalysis and active site [35]. Therefore, the selection of enzymes was based on taxonomic divergence, molecular weight and their reported enantioselectivity of common ketones in the literature. Six ketoreductases belonging to Archaea/ Bacteria/Eukarya namely, Sulfolobus sulfotaricus alcohol dehydrogenase (ADH) (SsADH), SDRs Zygosaccharomyces rouxii SDR (ZRK) and Hansenula polymorpha DL-1 peroxisomal 2,4-dienoyl-CoA reductase (Hketo) and lastly, Corynebacterium strain ST-10 phenylacetaldehyde reductase (PAR), Synechococcus sp. PCC 7942 3-ketoacyl-[acyl-carrier-protein] reductase (FabG) and Bacillus sp. ECU0013 ADH (ByeuD) were chosen, (highlighted in bold in Table 1). E. coli was selected as the organism to express the recombinant ketoreductases given its ease of growth to obtain large biomass and well-understood physiology. The enzymes were over-expressed using the T7 expression system consisting of the respective ketoreductase-pET28a plasmids and the E. coli BL21(DE3) strain. It is essential for the proteins to be soluble in vivo for efficient whole-cell catalysis. Therefore, the expression of the enzyme was verified by isolating the Histagged proteins from cytosolic fractions and visualizing on a Coomassie stained SDS-PAGE (Fig. 1). The overexpression of the desired ketoreductase also helps reduce side reactions from other competing enzymes in the bacterial cell.

\section{ZRK shows the best activity towards a variety of ketones amongst the compared ketoreductases}

Six ketoreductases are compared for catalyzing three pro-pharmaceutical chiral compounds 3,6 and 8 as substrates in Table 2. SsADH, PAR and ByeuD that converted below $30 \%$ for all substrates were deemed as low yielding reactions. FabG and Hketo showed moderate

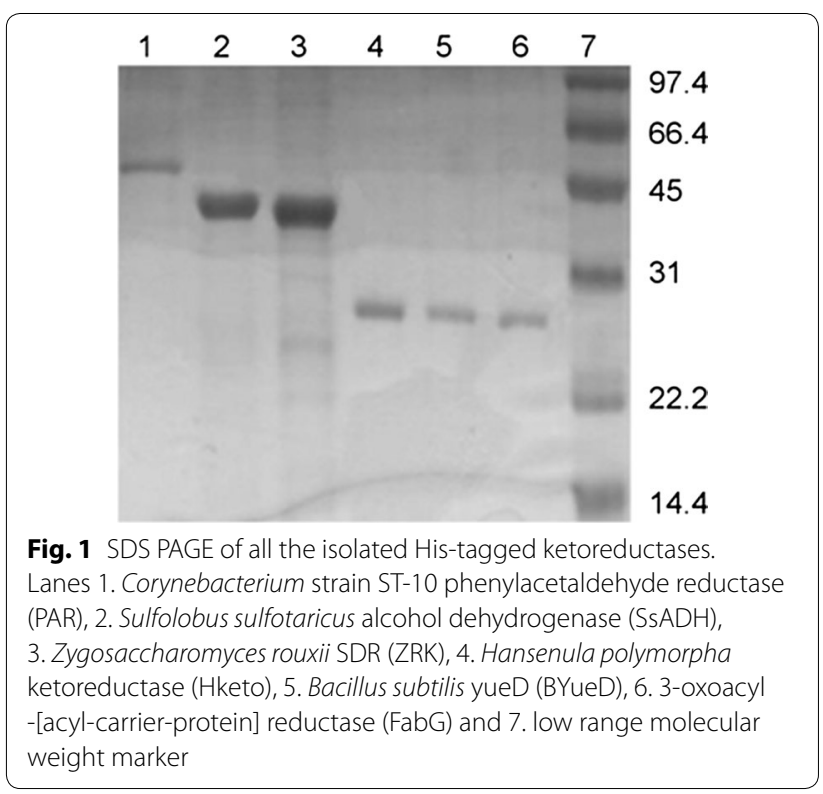


Table 2 Comparison of alcohol yields for commercially relevant ketones using six ketoreductases

\begin{tabular}{lllccc}
\hline Prochiral ketone & \multicolumn{2}{l}{ Alcohol yield (\%) } & & \\
\cline { 2 - 6 } & SsADH & ByeuD & FabG & Hketo & PAR \\
\hline Aprepitant ketone intermediate (compound 3) & 0.8 & 7 & 0 & 1.32 & 0 \\
Sitagliptin ketone intermediate (compound 8) & 9.6 & 29 & 38 & 87 & 22.6 \\
Dolastatin ketone intermediate (compound 6) & 2.98 & 26 & 31.8 & 41 & 18 \\
\hline
\end{tabular}

ZRK was found to be the best catalyst among the chosen enzymes. The substrates used are as follows, aprepitant ketone intermediate: 1-(3,5-bis-trifluoromethylphenyl)-ethanone, Sitagliptin ketone intermediate: 3-oxo-4-(2,4,5-trifluoro-phenyl) butyric acid methyl ester and dolastatin ketone intermediate: 2-phenyl-1-thiazol-2yl-ethanone

conversions, $30-60 \%$ of Sitagliptin intermediate (3-oxo4-(2,4,5-trifluoro-phenyl) butyric acid methyl ester) and Dolastatin intermediate (2-phenyl-1-thiazol-2-yl-ethanone). However, its yield was less than $2 \%$ in the case of Aprepitant intermediate [1- (3,5-Bis-trifluoromethylphenyl)-ethanone]. ZRK stands out with $>98 \%$ yield for Sitagliptin and Dolastatin intermediates and 22\% for the Aprepitant intermediate. HPLC analysis revealed an excess of $(S)$-specific alcohol for the three ketones, data for ZRK shown in Table 3. It should be noted that the Aprepitant intermediary of interest is an $(R)$-enantiomer but the enzyme produces an $(S)$-isomer and hence has been included in the list of substrates. We hypothesised that there underlies a specific mode of substrate binding that results in (S)-specific conversion. Elucidating the mode of substrate binding could help exploit the ketoreductases for obtaining specific chiral synthons. The most efficient enzyme in the current study i.e., ZRK, was selected for evaluation by in silico modelling and docking experiments.

\section{Sorbitol is a preferred carbon source compared to glucose for cofactor enrichment}

During biotransformation, E. coli has a finite concentration of $\mathrm{NAD}(\mathrm{P}) \mathrm{H}$, which would restrict the conversion of the substrate ketone. Regeneration of cofactors is essential for high catalytic potential [36]. In-vitro enzyme mediated cofactor regeneration methods employ either an enzyme or substrate coupled approach. These strategies typically use glucose/ formate dehydrogenase to catalyze the reduction of $\mathrm{NAD}(\mathrm{P})$ [37]. A non-genetic means of enhancing cofactors is by modifying the external environment by using carbon sources with a lower oxidation state [38]. Glucose, the commonly used carbon source for fermentation has an oxidation state of 1 . Other carbon sources like xylose, fructose and sorbitol have an oxidation state of $0,+1$ and -1 respectively [39]. Sorbitol with the lowest oxidation state would be the preferred carbon source to be evaluated.

In bacteria, sorbitol metabolism produces NADH using two separate pathways. It is catalyzed by sorbitol dehydrogenase $(S d h)$ to form fructose or as in E. coli, the sorbitol permease complex $(S r l A / B / E)$ helps in the uptake and phosphorylation of sorbitol into sorbitol-6-phosphate. Subsequently, it is oxidized by sorbitol-6-phosphate dehydrogenase $(S r l D)$ into fructose-6-phosphate [40, 41] with a concomitant release of NADH. This fructose/ fructose-6-phosphate proceeds towards the glycolytic pathway (Fig. 2). We hypothesize that this additional $\mathrm{NADH}$ enhances the pool of cofactors available for the ketoreductases to convert the ketone to their respective alcohol. NADH and NADPH are inter-converted during cellular energy deficit by nadK (NAD Kinase) which converts $\mathrm{NAD}^{+}$into $\mathrm{NADP}^{+}$. It is subsequently reduced to NADPH by the membrane bound or soluble pyridine nucleotide transhydrogenase $(P n t A / B)$ [42] as illustrated in Fig. 2. Enhanced alcohol production was observed in cells utilizing sorbitol instead of glucose as the carbon source during biotransformation (Table 3).

Table 3 Sorbitol is better than glucose as carbon source

\begin{tabular}{llllr}
\hline Ketone intermediate & \multicolumn{2}{l}{ Alcohol yield \% } & & \\
\cline { 2 - 5 } & PBS & PBS with glucose & PBS with sorbitol & (S)-enantiomer \\
\hline Talampanel ketone intermediate (compound 1) & 1.25 & 79 & 87 & 90 \\
MA-20565 ketone intermediate (compound 5) & 2.75 & 37 & 91 & 100 \\
Dolastatin ketone intermediate (compound 6) & 5 & 23 & 100 & 96 \\
\hline
\end{tabular}

Whole-cell E. coli expressing ZRK in sorbitol containing media gives higher alcohol yields than in glucose (average yields from three experiments reported). The substrates used are as follows, talampanel ketone intermediate: 3,4-methylenedioxyphenyl acetone, MA-20565 ketone intermediate: 3-trifluoromethyl acetophenone and dolastatin ketone intermediate: 2-Phenyl-1-thiazol-2-yl-ethanone 


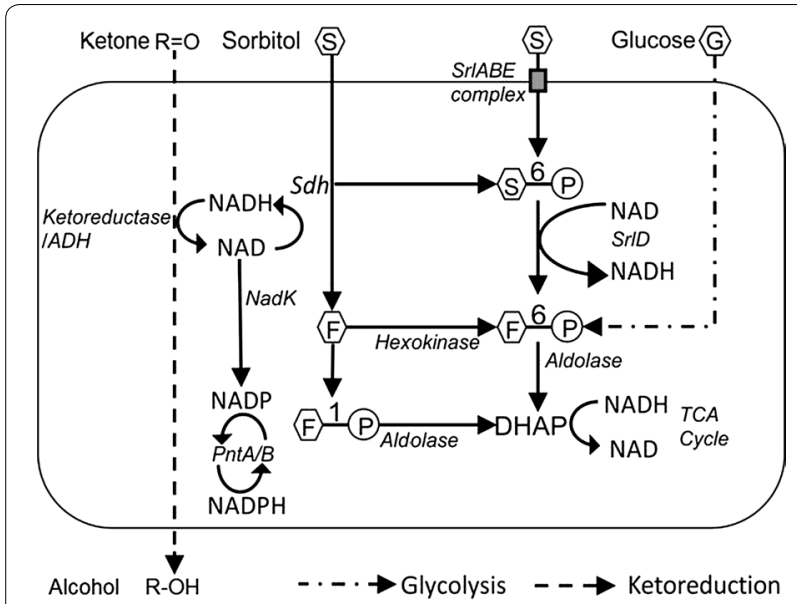

Fig. 2 Sorbitol metabolism in E. coli. A model illustrating the biochemical pathways involved in the production of additional reduced cofactors $\mathrm{NAD}(\mathrm{P}) \mathrm{H}$ by introduction of sorbitol as the carbon source instead of glucose in whole-cell biotransformation

In silico ZRK model with other relevant ketones showed a potential (S)-enantiomer producing enzyme

Homology modelling and molecular docking provide reasonable information about substrate interaction with the catalytic site and also help deduce reasons for stereo-selectivity [43-45]. Some of these studies incorporate stereochemistry rules such as Prelog and antiPrelog [44] and state that combining these rules with binding orientations provide high success in predicting the product enantiomer [44]. Understanding the mode of substrate binding could help us exploit these enzymes for obtaining specific product enantiomers. To discern the high $(S)$-enantiomeric excess (\%EE), we considered using similar methods in probing the substrate binding mode and mechanism of ZRK for further application to other ketones.

\section{Homology modelling of ZRK}

ZRK was modelled using yeast methyl glyoxal/isovaleraldehyde reductase Gre2 [PDB ID: 4PVD] [45] as a template that has $44 \%$ homology (Fig. 3a) using SWISSMODEL. PROCHECK was used to validate the $3 \mathrm{D}$ structure; it showed $\sim 88 \%$ of the residues lie in the most favored region (Fig. 3b) compared to $~ 91 \%$ residues in the template (Fig. 3c). The Root Mean Square Deviation (RMSD) between C $\alpha$ atoms of the template and the target structures was $0.7 \AA$. Structural and sequential information, by comparison, showed the catalytic triad serine, tyrosine and lysine (important for ketone to alcohol reduction) [46] was conserved in the ZRK model.
Substrate binding mode evaluation shows (S)-enantiomer oriented reduction

Molecular docking was used to predict substrate binding to the ZRK model [47]. Additional pharmaceutically relevant ketones (compounds 1, 2, 4, 5 and 7 from Table 4) along with previously catalyzed compounds (compounds 3, 6 and 8 from Table 2) were docked to predict substrate binding and enantiomer production. The binding orientations projected for four out of eight substrate molecules are shown in Fig. 4. Dock poses revealed that the carbonyl functional group connected to the carbon atoms on either side prefers to form hydrogen bond interactions with two (S127 and Y165) out of three catalytic triad residues. The carbonyl oxygen of compound 1 interacts with catalytic residues and the aromatic portion is positioned perpendicular to nicotinamide moiety of NADPH (Fig. 4a). In such an orientation, methylene dioxyphenyl ring forms hydrophobic interactions with substrate binding pocket residues such as Y197, F214 as well as the catalytic residue Y165. In the case of compound 2, the carbonyl group present on pyrolidinone ring interacts with catalytic residues while the phenyl ring of CBZ group forms hydrophobic interactions with surrounding residues (Fig. 4b). The carbonyl group of substituted acetophenone (Fig. 4c, d) establishes hydrophilic interactions similar to compound 1 and 2 whereas the metasubstituted phenyl ring forms hydrophobic interactions. The docking results of the remaining compounds were similar to the above observations.

\section{Molecular dynamics simulations confirm the docking evaluation}

We performed molecular dynamics simulations and binding pose of compound 1 (Fig. 4a) with modeled ZRK. The NADPH molecule coordinates acquired from the template structure by superposition method are included in ZRK-compound 1 complex simulation. The MD simulation analyses such as RMSD, interaction energies with cofactor and substrate as well as hydrogen bonding analyses (Additional file 1: Fig. S1) revealed that docking predicted binding mode is reasonable. The interaction pattern of substrates with catalytic residues S127 and Y165 established in the current study was consistent with earlier report [47] which is a prerequisite for further steps of the catalytic mechanism. Time-dependent backbone RMSD showed that the simulations were stable with no observable artifacts. The average RMSD value for $\mathrm{ZRK}+\mathrm{NADPH}+$ compound 1 complex oscillated around $0.2 \mathrm{~nm}$ (Additional file 1 : Fig. S1). Further, inter-molecular hydrogen bond interaction analyses between ZRK and NADPH revealed 10-12 hydrogen bond interactions were possible and 


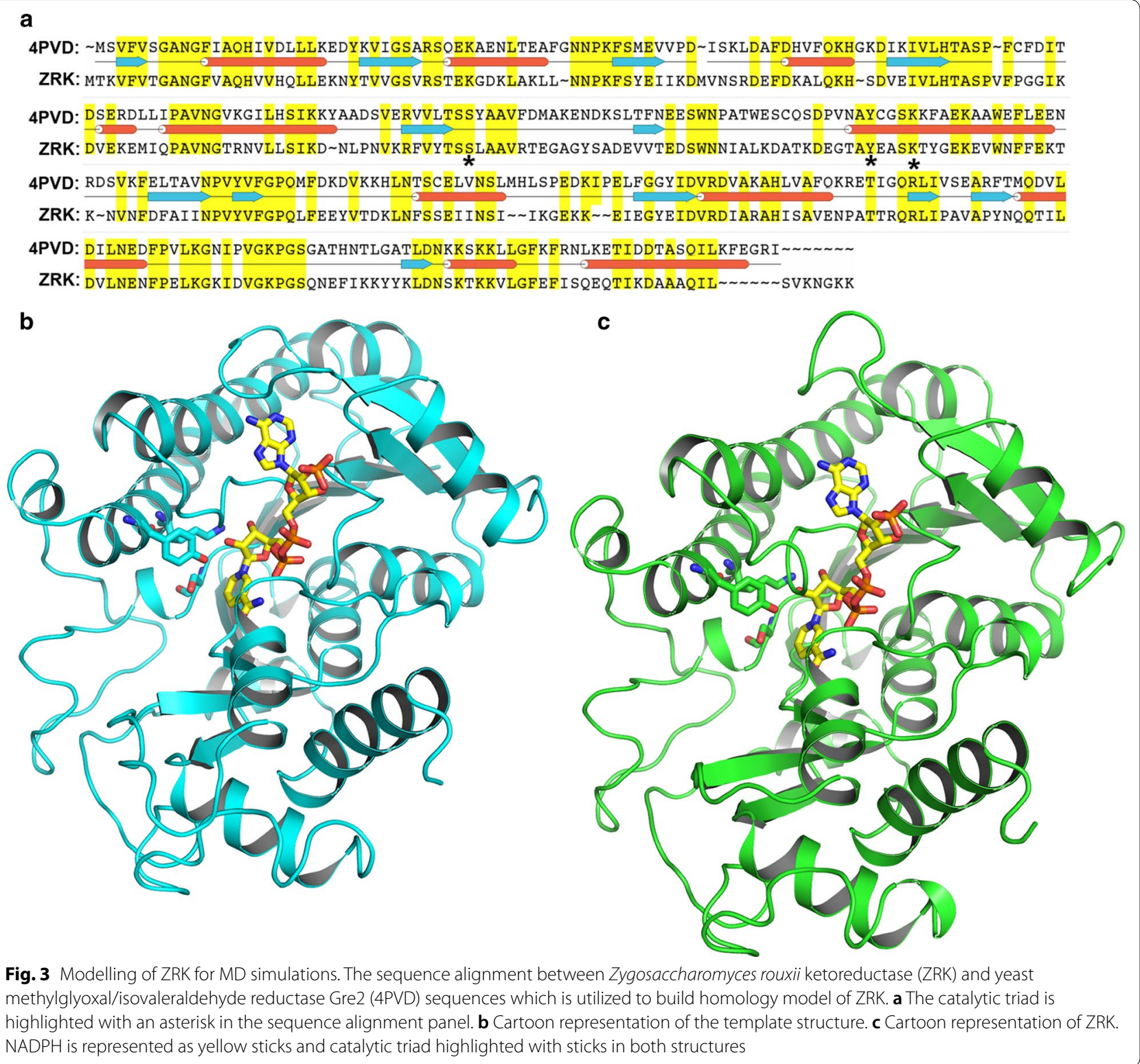

consistent throughout $10 \mathrm{~ns}$ simulation. The two hydrogen bonds predicted by molecular docking between ZRK and substrate molecule (compound 1) were also preserved during the time course of simulation (Additional file 1: Fig. S1b). The short range (SR) columbic and Vander Waals interaction energies are calculated (Additional file 1: Fig. S1c, d) for substrate and NADPH with ZRK. The analyses also support the stability of the simulations and reveal that NADPH interacts firmly with ZRK than the substrate. This is anticipated as NADPH makes large surface area contact with ZRK comprising of charged (predominantly) and hydrophobic portions.
Molecular editor tools incorporated in Maestro suite were utilized to edit the carbonyl of compound 1 initially to methylene $(\mathrm{CH} 2)$ as shown in Additional file 1: Fig. S2b. Hydroxyl groups were substituted at methylene hydrogen atoms to generate models of enantiomeric alcohols (see Additional file 1: Fig. S2c, d). The NADPH is replaced with $\mathrm{NADP}^{+}$for better understanding and visual inspections. The hydroxyl that faces $\mathrm{NADP}^{+}$has $\mathrm{S}$ configuration $[\mathrm{S}-\mathrm{OH}]$ and hydroxyl away from cofactor, facing A129 residue has R-configuration. The hydroxyl group in R-configuration does not have enough room and is sterically crowded; it may lead to a steric clash with A129, Y165 and hydroxyl group of S127. In the case of 


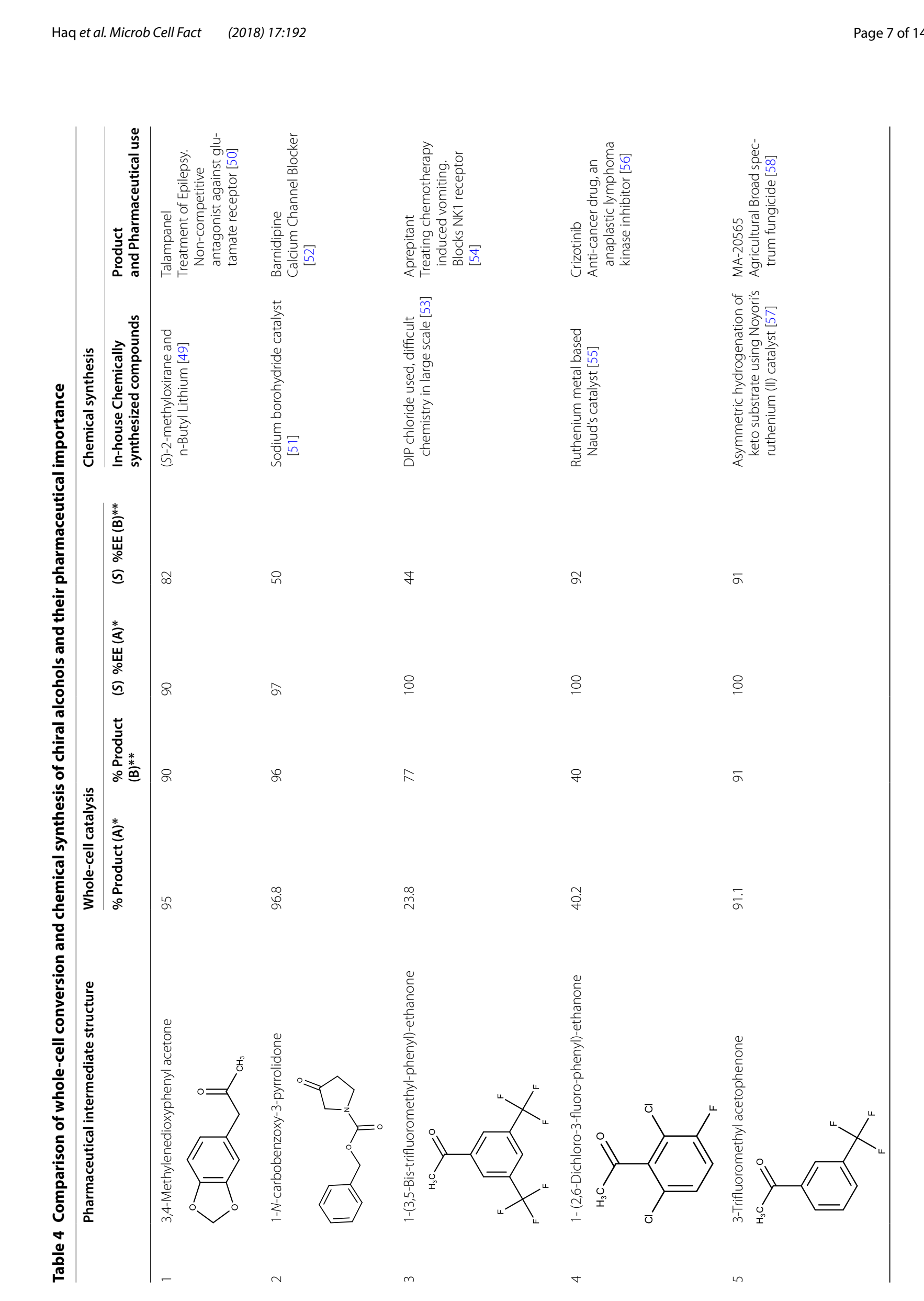




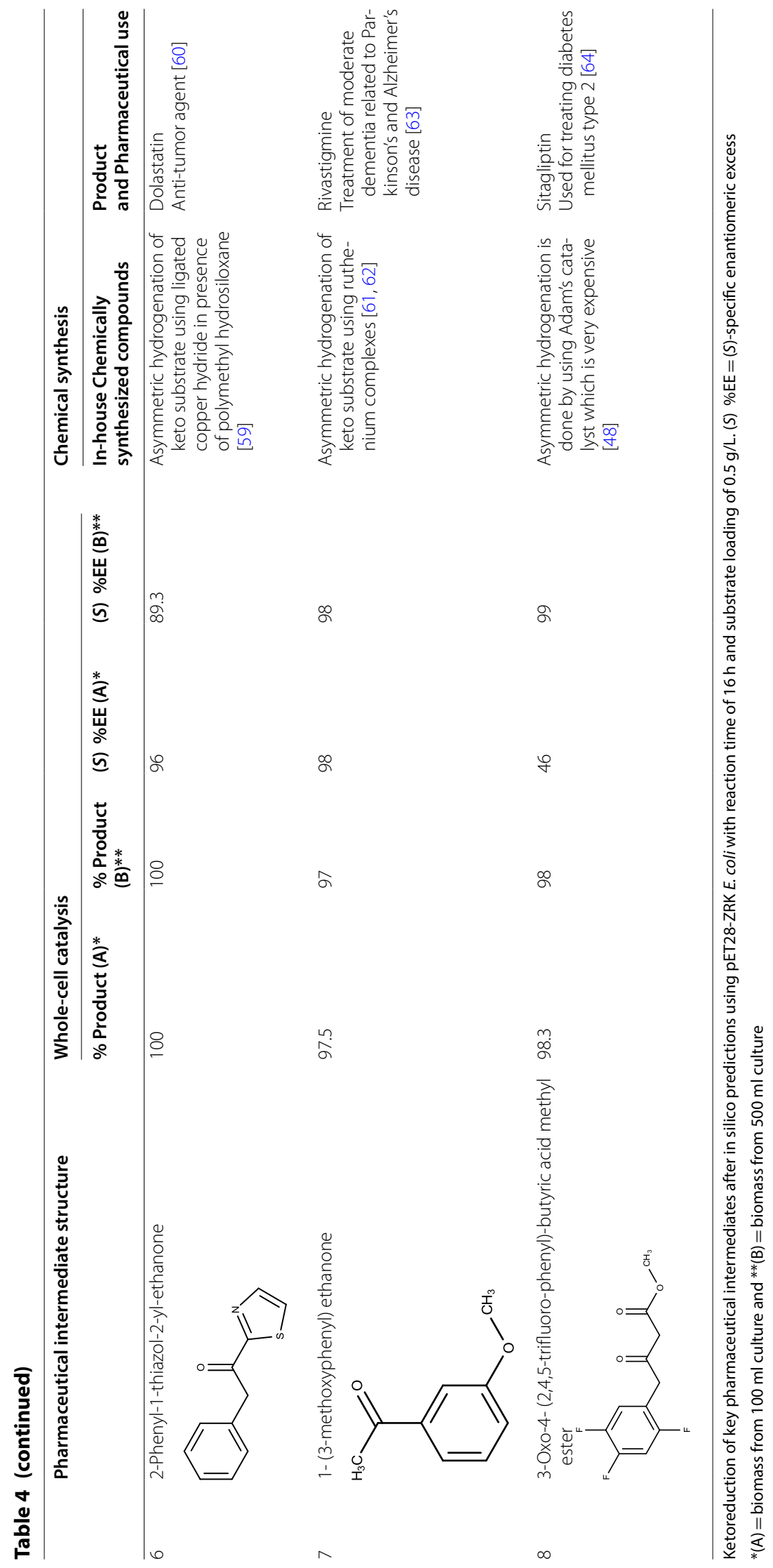




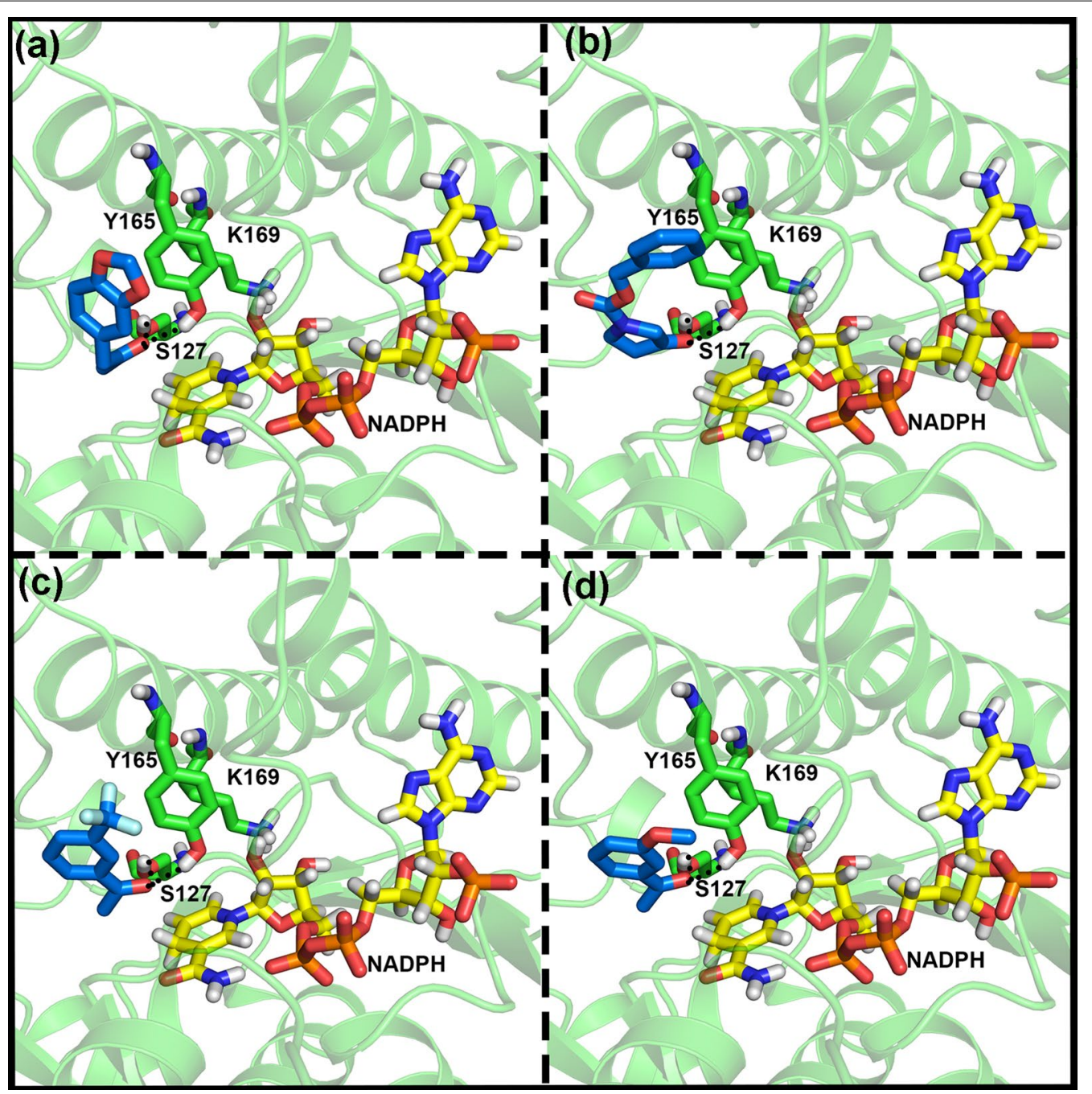

Fig. 4 Binding mode of substrate moleucles. Molecular docking predicted binding interaction (depicted in dashed black lines) modes for compound $1 \mathbf{a}$, compound $2 \mathbf{b}$, compound $3 \mathbf{c}$, and compound $7 \mathbf{d}$. Substrates are represented with blue sticks, catalytic residues and NADPH are shown as green and yellow sticks respectively. Hydrogen bonds between substrate and triad residues are depicted with broken lines

alternative $(S)$-stereomer, hydroxyl group has enough room to adjust as the location is open as shown in Additional file 1: Fig. S2c. This could be the reason for the substrates (compound 1-7) evaluated in the current study to yield higher amounts of $(S)$-configuration products. Binding orientations of the substrate revealed that the reface of the substrate toward NADPH results in the formation of the $(S)$-enantiomer of the alcohols for all of the studied compounds except compound 8. Docking studies of compound 1-7 clearly demonstrated that the substrate binding orientations are pro- $(\mathrm{S}) / \mathrm{Si}$ and consistent with other ketoreductases that yield $(S)$-enantiomers. It is well-known that the stereo-specificity of the enzymecatalyzed keto reductions can be predicted with Prelog's rules, which depends on the steric situation of substrates
[48]. Noticeably, the steric terms of the substrate with catalytic site residues and NADPH dictate the orientation and further stereo-selectivity of the product. Observations of our current results revealed that NADPH cofactor is bound in the syn conformation, which exposes the $\mathrm{Si}$ hydrogen atom of reduced nicotinamide toward the enzyme active site as a reactant for ketone reduction [3]. This understanding helped in generating 90\% (S)-enantiomeric excess of other important pro-pharmaceutical ketones. It is interesting to note that Sitagliptin ketone intermediate (compound 8) did not concur with the predictions. All the compounds except the compound 8 [3-Oxo-4-(2,4,5-trifluoro-phenyl)-butyric acid methyl ester] yielded $(S)$-enantiomers. Examination of the binding orientation of compound 8 revealed the possibility of 

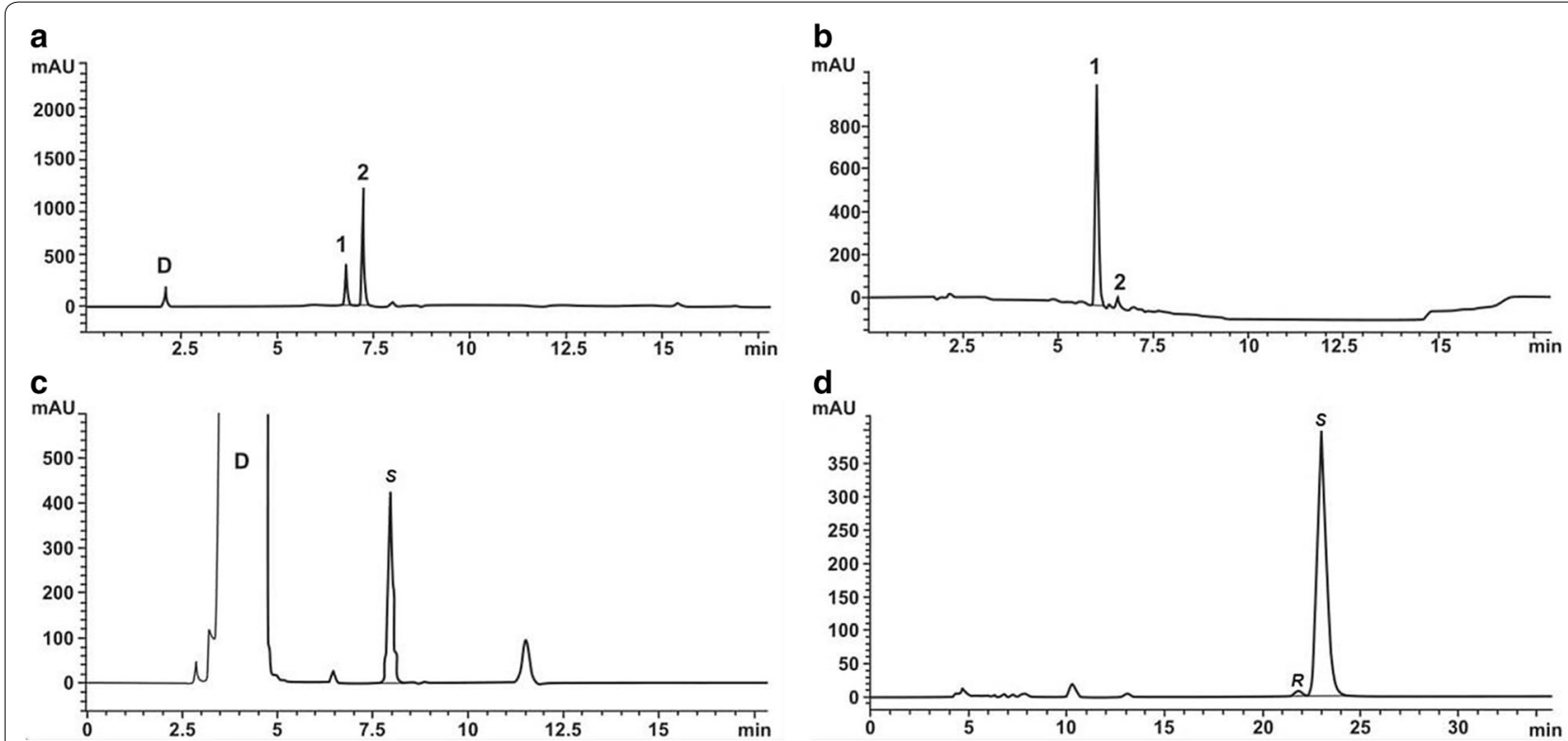

Fig. 5 Chromatograms depicting bioconversion of pro chiral ketones. HPLC analysis of whole-cell transformed Crizotinib ketone intermediate (a) and the chiral separation of its alcohol (b). Similarly, whole-cell transformed dolastatin ketone intermediate (c) and the chiral separation of its alcohol (d). 1: alcohol product, 2: substrate, D: DMSO, S: (S)-isomer and R: (R)-isomer. In both the cases, the (S)-isomer production is $>90 \%$

variant binding modes (see Additional file 1: Fig. S3a \& S3c). The compound 8 binding orientation is like compound 1 with the hydroxyl group towards NADPH but is in R-configuration which is contrary to the orientation of the other compounds. This difference might be arising due to differences in chemical structure, for instance, flexible linkers connected to the carbonyl group (reaction center). In the case of compound 3, the product of commercial interest is the $(R)$-enantiomer but the enzyme converts it to an $\mathrm{S}$-alcohol as predicted by the modelling study.

\section{Evaluation of the in silico results by using ZRK expressing cells}

A $500 \mathrm{ml}$ scale ketoreduction reaction as outlined in the experimental section was set up for 8 substrates (compounds 1-8 from Table 4) [48-64] that are key intermediates in the syntheses of different therapeutic molecules. These trials were used to isolate the alcohol product in sufficient amounts and carry out a preparative chiral HPLC and determine the nature of the isomer produced. We observe that the $(S)$-enantiomer is preferentially produced during ketoreduction by ZRK and the yields are listed again in Table 4. Chromatograms for Crizotinib and Dolastatin intermediates featuring $(S)$-enantiomeric yield are shown in Fig. 5. Chromatograms for the rest of the substrates are provided in Additional file 1: Fig. S5S28. All the compounds except the Sitagliptin ketone intermediate (compound 8) yielded $(S)$-enantiomers.

\section{Conclusions}

Amongst the six evaluated ketoreductases, ZRK converts a diverse range of pharmaceutically important prochiral ketones. The mechanism contributing to enantioselectivity towards the production of $(S)$-alcohols has been elucidated by docking and MD simulations with a focus on active site architecture. The computational protocol optimized here can be useful to predict novel substrates that can bind to ZRK and undergo ketone to alcohol conversion. Regeneration of cofactors for biotransformation is usually enabled by either substrate coupled or enzyme coupled reactions [65]. To achieve similar results we used nutrient modulation, cofactor enhancement was attained by switching the carbon source from glucose to sorbitol. It eliminates the need for co-expressing another enzyme that impacts the energy resources of the cell, the same would now be available for chiral conversion. This understanding of the catalytic mechanism and cofactor augmentation can be extended to other ketoreductases and substrates to reduce the enzyme screening space and enable manufacture of high value chiral alcohols.

\section{Methods}

Reagents and chemicals

Escherichia coli strains DH5 $\alpha$ (Novagen, U SA) and BL21(DE3) (Novagen, USA) are used as hosts for subcloning and over-expression respectively. Restriction enzymes NdeI and XhoI are purchased from NEB (New England Biolabs). A set of high value prochiral 
ketone intermediates namely, 3,4-methylenedioxyphenyl acetone, 1-N-carbobenzoxy-3-pyrrolidione or 3-hydroxy-pyrrolidone-1-carboxylicacid benzyl ester, 1-(3,5-bis-trifluoromethyl-phenyl)-ethanone, 1(2,6-dichloro-3-fluoro-phenyl)-ethanone, 3-trifluoromethyl acetophenone, 2-phenyl-1-thiazol-2-yl-ethanone, 1- (3-methoxyphenyl) ethanone, 3-oxo-4- (2,4,5-trifluoro-phenyl)-butyric acid methyl ester as important intermediates in various pharmaceutical syntheses are synthesized as outlined in Additional file 1. Corresponding racemic alcohols are synthesized from respective ketones using the sodium borohydride method reported elsewhere [49].

\section{Construction of the different ketoreductase expression vectors and transformation into BL21(DE3)}

Genes encoding the six enzymes from diverse taxa i.e., Sulfolobus sulfotaricus alcohol dehydrogenase (ADH) from archaea (Ssadh), fungal SDRs Zygosaccharomyces rouxii SDR (ZRK) and Hansenula polymorpha DL-1 peroxisomal 2,4-dienoyl-CoA reductase (Hketo) and lastly, Corynebacterium strain ST-10 phenylacetaldehyde reductase (PAR), Synechococcus sp. PCC 7942 3-ketoacyl[acyl-carrier-protein] reductase (FabG) and Bacillus sp. ECU0013 ADH (ByueD) from bacteria are synthesized using optimal codon usage for E. coli (Geneart, http:// www.geneart.com). The gene sequences are deposited in DDBJ with the following accession numbers (LC325171 for SsADH, LC325172 for ZRK, LC325173 for PAR, LC325174 for ByeuD, LC325175 for Hketo, LC325176 for FabG). These are then sub-cloned into pET28a vector (Novagen) using the restriction sites NdeI and XhoI with an N-terminal His-tag in frame. Soluble protein expression experiments are performed as in the protocol followed for whole-cell transformation. The cells are harvested $18 \mathrm{~h}$ post induction and the soluble Histagged ketoreductases are purified by $\mathrm{Ni}-\mathrm{NTA}$ affinity chromatography.

\section{Whole-cell biotransformation using $E$. coli expressing various ketoreductases}

Over-expression of the enzymes using the different pET28a-ketoreductase plasmids is done in $E$. coli BL21(DE3) strain using standard procedures [66]. In brief, the pET28a-ketoreductase plasmid transformed BL21 (DE3) cells are grown in $100 \mathrm{ml} \mathrm{LB-broth} \mathrm{with}$ $50 \mu \mathrm{g} / \mathrm{ml}$ Kanamycin at $37^{\circ} \mathrm{C}$ to an OD of 0.6 , followed by induction with $200 \mu \mathrm{M}$ IPTG and continued incubation at $18^{\circ} \mathrm{C}$ for $16 \mathrm{~h}$. The cells are harvested and resuspended in $10 \mathrm{ml}$ phosphate buffered saline (PBS) containing $10 \%$ carbon source $(w / v)$, either phosphate buffered saline
(PBS), PBS + glucose or PBS + sorbitol. The cells are acclimatized for $2 \mathrm{~h}$ to use the provided carbon source before adding the prochiral ketone substrates to the suspension at $0.5 \mathrm{~g} / \mathrm{L}$ concentration. This culture and ketone suspension is incubated at $30^{\circ} \mathrm{C}$ for $16 \mathrm{~h}$ to allow the cells to perform the biotransformation. To determine the chiral alcohol yield and EE \%, the cell suspension is extracted with an equal volume of Ethyl acetate and the organic layer is subjected to HPLC analysis. E. coli BL21(DE3) cells over-expressing the different ketoreductases are grown overnight at $37^{\circ} \mathrm{C}$ in $500 \mathrm{ml} \mathrm{LB}$ followed by induction with IPTG and incubation as above. The cells are harvested and resuspended in $500 \mathrm{ml}$ of PBS with $10 \%$ sorbitol $(\mathrm{w} / \mathrm{v})$ before continuing the above procedure. This is done to compare the consistency in production of chiral synthons by ZRK at a higher volume.

\section{Analytical methods}

The conversion and enantiomeric excess of the wholecell biotransformation is monitored using HPLC. A Shimadzu LC-2010CHT HPLC equipped with Photodiode Array detector set at $205 \mathrm{~nm}$ is utilized. Either acetonitrile and $0.1 \%$ trifluoroacetic acid or acetonitrile and $0.05 \%$ perchloric acid in water is delivered to a $150 \mathrm{~mm} \mathrm{X}$ $4.6 \mathrm{~mm}, 5 \mu \mathrm{m}$, Zorbax SB-CN, column at a rate of $1 \mathrm{~mL} /$ min. Normal phase HPLC is carried out to determine the enantiomeric excess of the isomers produced. The mobile phase, $2 \% \mathrm{n}$-hexane and $98 \% n$-hexane: ethanol (50:50) is delivered to a Chiralcel OZ-H 4.6 X $250 \mathrm{~mm}$, $5 \mu \mathrm{m}$, at a rate of $0.7 \mathrm{~mL} / \mathrm{min}$. The racemic mixture generated by sodium borohydride method (from the prochiral ketones) is separated into $(R)$ or $(S)$ isomers by using preparative chiral HPLC techniques and their retention time (RT) value is fixed as a standard for determining the enantiomeric excess of the isomers produced following the ketoreduction.

\section{Homology model generation for ZRK}

Zygosaccharomyces rouxii SDR (ZRK: Swiss-Prot code Q9UV57 homologs are identified using Protein BLAST and all non-redundant proteins as search set on the NCBI database. The best hit is used as a template for homology modelling using SWISS-MODEL webserver [67]. The template structure selected by blastp search is specified explicitly to generate homology models which are validated by PROCHECK software $[68,69]$.

\section{Binding mode determination of substrate molecules by molecular docking}

Eight pro-chiral pharmaceutically important substrates (Nos. 1-8 from Table 4) are considered in catalytic 
site docking to determine binding orientations. The ligands are converted to 3D using LigPrep of Schrodinger suite which performs tasks such as protonation at specified $\mathrm{pH}$, generation of tautomers, ionization states, and probable stereomers [70]. The output is considered for multi-conformation generation by Macromodel [71] module which uses Monte Carlo method of exploring torsional space. OPLS_2005 force field is used and energy minimization is done in 500 steps using TNCG method (Energy window is $21 \mathrm{~kJ} / \mathrm{mol}$ which is default value). The redundancy threshold is $0.5 \AA$ RMSD, and redundant conformers are removed. The maximum number of conformers to be generated is set to 25. Default parameters are used for the remaining options. Glide v5.5 docking module (in Schrodinger suite) is employed to predict the binding orientations for the eight substrates molecules [72]. Glide-SP algorithm uses pre-computed grids generated using receptor sites defined by the centroids of the bound ligands/ selected atoms. The lowest energy poses are then subjected to a Monte Carlo procedure that sample nearby torsional minima. The compounds are then ranked using GlideScore that includes terms for steric clashes and buried polar groups. Default van der Waal's scaling is used (1.0 for the receptor and 0.8 for the ligand). Advanced settings are edited to increase the pose sampling. Total 10,000 poses (default 5000) per ligand are set for the initial phase of docking and poses per ligand per energy minimization raised to 1000 from 400 . Total ten poses per ligand are saved as output for post docking analyses.

\section{Molecular dynamics simulations}

MD simulations are performed using GROMACS program (version 4.5.4) with the Amber99SB force field [73]. AM1-BCC charges are computed for substrates with the Antechamber software [74]. For MD simulations, each system is inserted in a water box ( $\mathrm{pH} 7.0)$ of TIP3P water, which extended at least $12 \AA$ away from any given protein atom. All systems are neutralized by adding counter ions and replacing the overlapping solvent molecules. Steepest descent algorithm is used for energy minimization. Position restrained MD run performed for 200 ps during heating step ( $300 \mathrm{~K}$ ) so that solute molecules (protein and ligand) molecules are restrained whereas water molecules with counter ions are allowed to move and equilibrate. The final simulation is performed for $10 \mathrm{~ns}$ with a 2 -fs time step. Temperature and pressure are maintained at $300 \mathrm{~K}$ and $1 \mathrm{~atm}$ respectively using the $\mathrm{v}$-rescale temperature and Parrinello-Rahman pressure coupling method.

\section{Additional file}

Additional file 1. Supplementary Information.

\section{Abbreviations}

E. coli: Escherichia coli; HPLC: high-performance liquid chromatography; $\mathrm{OD}_{600}$ : optical density at the wavelength of $600 \mathrm{~nm}$; \%EE: enantiomeric excess percentage; RMSD: Root Mean Square Deviation; PBS: phosphate buffered saline; NAD: Nicotinamide Adenine Dinucleotide; NADP: Nicotinamide Adenine Dinucleotide Phosphate; MD: molecular dynamics.

\section{Authors' contributions}

SD conceived the study. APS and SFH performed all the primary and final experiments. SK and IH assisted with the execution of bio-processes designed by KT, AA, SS, SS and NK. The data was analyzed by KT, AA, SS, SFH and SS. RS provided support via the grant for APS and NK as well as the infrastructure for computational studies performed by NB. SS, SS, APS, NB and NK wrote the manuscript. RS and SD revised the manuscript. All authors read and approved the final manuscript.

\section{Author details}

${ }^{1}$ Anthem Biosciences Pvt. Ltd, Bengaluru, India. ${ }^{2}$ Bugworks Research India, Pvt. Ltd, Bengaluru, India. ${ }^{3}$ Centre for Pharmaceutical Biotechnology, University of Illinois Chicago, Chicago, USA. ${ }^{4}$ Shasun Research Center, Chennai, India. ${ }^{5}$ Institute for Stem Cell Biology and Regenerative Medicine, Bengaluru, India.

${ }^{6}$ Centre for Cellular and Molecular Platforms, Bengaluru, India. ${ }^{7}$ Department of Biophysics, Molecular Biology and Bioinformatics, University of Calcutta, Kolkata, India. ${ }^{8}$ PerkinElmer, Bengaluru, India.

\section{Acknowledgements}

Not applicable.

\section{Competing interests}

The authors declare that they have no competing interests as defined by Nature Publishing Group, or other interests that might be perceived to influence the results and/or discussion reported in this paper.

\section{Availability of data and materials}

The data related to sequences has been deposited in DDBJ with the Accession Numbers LC325171-LC32516. The datasets used and/or analyzed during the current study are available from the corresponding author on request.

\section{Consent for publication}

Not applicable.

Ethics approval and consent to participate

Not applicable.

\section{Funding}

The financial support from Biotechnology Industry Partnership Program, Department of Biotechnology, India (DBT Sanction Order No: BT/ BIP0475/13/11) and Grant BT/PR13429/BRB/10/767/2009 to Prof. S. Ramaswamy is gratefully acknowledged.

\section{Publisher's Note}

Springer Nature remains neutral with regard to jurisdictional claims in published maps and institutional affiliations.

Received: 16 May 2018 Accepted: 21 November 2018

Published online: 03 December 2018

\section{References}

1. Faber K. Introduction and background information. biotransformations org chem. Berlin: Springer; 2011. p. 1-30. http://link.springer. com/10.1007/978-3-642-17393-6_1. 
2. Kataoka M, Kita K, Wada M, Yasohara Y, Hasegawa J, Shimizu S. Novel bioreduction system for the production of chiral alcohols. Appl Microbiol Biotechnol. 2003;62:437-45.

3. Woodley JM. New opportunities for biocatalysis: making pharmaceutical processes greener. Trends Biotechnol. 2008;26:321-7.

4. Goldberg K, Schroer K, Lütz S, Liese A. Biocatalytic ketone reduction-a powerful tool for the production of chiral alcohols-part I: processes with isolated enzymes. Appl Microbiol Biotechnol. 2007;76:237-48.

5. Goldberg K, Schroer K, Lütz S, Liese A. Biocatalytic ketone reduction-a powerful tool for the production of chiral alcohols-part II: whole-cell reductions. Appl Microbiol Biotechnol. 2007;76:249-55.

6. Kratzer R, Pukl M, Egger S, Nidetzky B. Whole-cell bioreduction of aromatic alpha-keto esters using Candida tenuis xylose reductase and Candida boidinii formate dehydrogenase co-expressed in Escherichia coli. Microb Cell Fact. 2008;7:37.

7. Murphy AC. Metabolic engineering is key to a sustainable chemical industry. Nat Prod Rep. 2011;28:1406-25.

8. Soni P, Banerjee UC. Enantioselective reduction of acetophenone and its derivatives with a new yeast isolate Candida tropicalis PBR-2 MTCC 5158. Biotechnol J. 2006;1:80-5.

9. Blaser H-U, Malan C, Pugin B, Spindler F, Steiner H, Studer M. Selective hydrogenation for fine chemicals: recent trends and new developments. Adv Synth Catal. 2003;345:103-51.

10. Moore JC, Pollard DJ, Kosjek B, Devine PN. Advances in the enzymatic reduction of ketones. Acc Chem Res. 2007:40:1412-9.

11. Ishige T, Honda K, Shimizu S. Whole organism biocatalysis. Curr Opin Chem Biol. 2005;9:174-80.

12. Hölsch K, Havel J, Haslbeck M, Identification Weuster-Botz D. cloning, and characterization of a novel ketoreductase from the cyanobacterium Synechococcus sp. strain PCC 7942. Appl Environ Microbiol. 2008;74:6697-702.

13. Wang L-J, Li C-X, Ni Y, Zhang J, Liu X, Xu J-H. Highly efficient synthesis of chiral alcohols with a novel NADH-dependent reductase from Streptomyces coelicolor. Bioresour Technol. 2011;102:7023-8.

14. Kawano S, Yano M, Hasegawa J, Yasohara Y. Cloning and overexpression of an NADH-dependent alcohol dehydrogenase gene from Candida maris involved in (R)-selective reduction of 5-acetylfuro[2,3-c]pyridine. Biosci Biotechnol Biochem. 2011;75:2155-61.

15. Niefind K, Müller J, Riebel B, Hummel W, Schomburg D. The crystal structure of R-specific alcohol dehydrogenase from Lactobacillus brevis suggests the structural basis of its metal dependency. J Mol Biol. 2003;327:317-28.

16. Cao H, Mi L, Ye Q, Zang G, Yan M, Wang Y, et al. Purification and characterization of a novel NADH-dependent carbonyl reductase from Pichia stipitis involved in biosynthesis of optically pure ethyl (S)-4-chloro-3-hydroxybutanoate. Bioresour Technol. 2011;102:1733-9.

17. Ye Q, Yan M, Yao Z, Xu L, Cao H, Li Z, et al. A new member of the shortchain dehydrogenases/reductases superfamily: purification, characterization and substrate specificity of a recombinant carbonyl reductase from Pichia stipitis. Bioresour Technol. 2009;100:6022-7.

18. Ravin NV, Eldarov MA, Kadnikov WV, Beletsky AV, Schneider J, Mardanova ES, et al. Genome sequence and analysis of methylotrophic yeast Hansenula polymorpha DL1. BMC Genomics. 2013;14:837.

19. Yasohara Y, Kizaki N, Hasegawa J, Wada M, Kataoka M, Shimizu S. Molecular cloning and overexpression of the gene encoding an NADPHdependent carbonyl reductase from Candida magnoliae, involved in stereoselective reduction of ethyl 4-chloro-3-oxobutanoate. Biosci Biotechnol Biochem. 2000;64:1430-6.

20. Saliola M, Shuster JR, Falcone C. The alcohol dehydrogenase system in the yeast, Kluyveromyces lactis. Yeast Chichester Engl. 1990;6:193-204.

21. Ni Y, Li C-X, Ma H-M, Zhang J, Xu J-H. Biocatalytic properties of a recombinant aldo-keto reductase with broad substrate spectrum and excellent stereoselectivity. Appl Microbiol Biotechnol. 2011;89:1111-8.

22. Kita K, Nakase K, Yanase H, Kataoka M, Shimizu S. Purification and characterization of new aldehyde reductases from Sporobolomyces salmonicolor AKU4429. J Mol Catal B Enzym. 1999;6:305-13.

23. Costello CA, Payson RA, Menke MA, Larson JL, Brown KA, Tanner JE, et al. Purification, characterization, cDNA cloning and expression of a novel ketoreductase from Zygosaccharomyces rouxii. Eur J Biochem FEBS. 2000;267:5493-501.
24. Chen Q, Hu Y, Zhao W, Zhu C, Zhu B. Cloning, expression, and characterization of a novel (S)-specific alcohol dehydrogenase from Lactobacillus kefir. Appl Biochem Biotechnol. 2010;160:19-29.

25. Timpson LM, Liliensiek A-K, Alsafadi D, Cassidy J, Sharkey MA, Liddell S, et al. A comparison of two novel alcohol dehydrogenase enzymes (ADH1 and $\mathrm{ADH} 2$ ) from the extreme halophile Haloferax volcanii. Appl Microbiol Biotechnol. 2013;97:195-203.

26. Ammendola S, Raia CA, Caruso C, Camardella L, D'Auria S, De Rosa $M$, et al. Thermostable NAD(+)-dependent alcohol dehydrogenase from Sulfolobus solfataricus: gene and protein sequence determination and relationship to other alcohol dehydrogenases. Biochemistry. 1992:31:12514-23.

27. Cannio R, Fiorentino G, Carpinelli P, Rossi M, Bartolucci S. Cloning and overexpression in Escherichia coli of the genes encoding NAD-dependent alcohol dehydrogenase from two Sulfolobus species. J Bacteriol. 1996;178:301-5.

28. Wang JC, Sakakibara M, Liu JQ, Dairi T, Itoh N. Cloning, sequence analysis, and expression in Escherichia coli of the gene encoding phenylacetaldehyde reductase from styrene-assimilating Corynebacterium sp. strain ST-10. Appl Microbiol Biotechnol. 1999;52:386-92.

29. Fox RJ, Huisman GW. Enzyme optimization: moving from blind evolution to statistical exploration of sequence-function space. Trends Biotechnol. 2008;26:132-8

30. Bisogno FR, Lavandera I, Kroutil W, Gotor V. Tandem concurrent processes: one-pot single-catalyst biohydrogen transfer for the simultaneous preparation of enantiopure secondary alcohols. J Org Chem. 2009;74:1730-2.

31. Shang Y-P, Chen Q, Kong X-D, Zhang Y-J, Xu J-H, Yu H-L. Efficient synthesis of (R)-2-chloro-1-(2,4-dichlorophenyl)ethanol with a ketoreductase from Scheffersomyces stipitis CBS 6045. Adv Synth Catal. 2017;359:426-31.

32. Ghatak A, Bharatham N, Shanbhag AP, Datta S, Venkatraman J. Delineating substrate diversity of disparate short-chain dehydrogenase reductase from Debaryomyces hansenii. PLOS ONE. 2017;12:e0170202.

33. Kallberg Y, Oppermann U, Persson B. Classification of the short-chain dehydrogenase/reductase superfamily using hidden Markov models. FEBS J. 2010:277:2375-86.

34. Kavanagh $\mathrm{KL}$, Jörnvall $\mathrm{H}$, Persson B, Oppermann U. Medium- and short-chain dehydrogenase/reductase gene and protein families: the SDR superfamily: functional and structural diversity within a family of metabolic and regulatory enzymes. Cell Mol Life Sci. 2008;65:3895-906.

35. Kallberg Y, Oppermann U, Jörnvall H, Persson B. Short-chain dehydrogenase/reductase (SDR) relationships: a large family with eight clusters common to human, animal, and plant genomes. Protein Sci Publ Protein Soc. 2002;11:636-41.

36. Kara S, Schrittwieser JH, Hollmann F, Ansorge-Schumacher MB. Recent trends and novel concepts in cofactor-dependent biotransformations. Appl Microbiol Biotechnol. 2014;98:1517-29.

37. Liu W, Wang P. Cofactor regeneration for sustainable enzymatic biosynthesis. Biotechnol Adv. 2007;25:369-84.

38. San K-Y, Bennett GN, Berríos-Rivera SJ, Vadali RV, Yang Y-T, Horton E, et al. Metabolic engineering through cofactor manipulation and its effects on metabolic flux redistribution in Escherichia coli. Metab Eng. 2002;4:182-92.

39. Lin H, Bennett GN, San K-Y. Effect of carbon sources differing in oxidation state and transport route on succinate production in metabolically engineered Escherichia coli. J Ind Microbiol Biotechnol. 2005;32:87-93.

40. Sola-Carvajal A, García-García MI, García-Carmona F, Sánchez-Ferrer Á. Insights into the evolution of sorbitol metabolism: phylogenetic analysis of SDR196C family. BMC Evol Biol. 2012;12:147.

41. Lengeler J. Nature and properties of hexitol transport systems in Escherichia coli. J Bacteriol. 1975;124:39-47.

42. Sauer U, Canonaco F, Heri S, Perrenoud A, Fischer E. The soluble and membrane-bound transhydrogenases UdhA and PntAB have divergent functions in NADPH metabolism of Escherichia coli. J Biol Chem. 2004;279:6613-9.

43. Dudzik A, Snoch W, Borowiecki P, Opalinska-Piskorz J, Witko M, Heider J, et al. Asymmetric reduction of ketones and $\beta$-keto esters by (S)-1-phenylethanol dehydrogenase from denitrifying bacterium Aromatoleum aromaticum. Appl Microbiol Biotechnol. 2015;99:5055-69.

44. Liang P, Qin B, Mu M, Zhang X, Jia X, You S. Prelog and anti-Prelog stereoselectivity of two ketoreductases from Candida glabrata. Biotechnol Lett. 2013:35:1469-73. 
45. Guo P-C, Bao Z-Z, Ma X-X, Xia Q, Li W-F. Structural insights into the cofactor-assisted substrate recognition of yeast methylglyoxal/isovaleraldehyde reductase Gre2. Biochim Biophys Acta. 2014;1844:1486-92.

46. Nambiar KP, Stauffer DM, Kolodziej PA, Benner SA. A mechanistic basis for the stereoselectivity of enzymic transfer of hydrogen from nicotinamide cofactors. J Am Chem Soc. 1983;105:5886-90.

47. He S, Ni D, Ma B, Lee J-H, Zhang T, Ghozalli I, et al. Ptdlns(3)P-bound UVRAG coordinates Golgi-ER retrograde and Atg9 transport by differential interactions with the ER tether and the beclin 1 complex. Nat Cell Biol. 2013;15:1206-19.

48. Prelog V. Specification of the stereospecificity of some oxido-reductases by diamond lattice sections. Pure Appl Chem. 1964;9. http://www.degru yter.com/view/j/pac.1964.9.issue-1/pac196409010119/pac196409010119 xml.

49. Wu G, Huang M. Organolithium reagents in pharmaceutical asymmetric processes. Chem Rev. 2006;106:2596-616.

50. Luszczki JJ. Third-generation antiepileptic drugs: mechanisms of action, pharmacokinetics and interactions. Pharmacol Rep. 2009;61:197-216.

51. Zhang W, Dai Y, Zhu H, Zhang W. One-pot synthesis of indene derivatives by CF3SO3H-promoted reactions of benzylic alcohols and 1,3-dicarbonyl compounds. Tetrahedron Lett. 2013;54:1747-50.

52. Parati G, Giglio A, Lonati L, Destro M, Ricci AR, Cagnoni F, et al. Effectiveness of barnidipine 10 or 20 mg plus losartan 50-mg combination versus losartan 100-mg monotherapy in patients with essential hypertension not controlled by losartan 50-mg monotherapy: a 12-week, multicenter, randomized, open-label, parallel-group study. Clin Ther. 2010;32:1270-84.

53. Liang J, Lalonde J, Borup B, Mitchell V, Mundorff E, Trinh N, et al. Development of a biocatalytic process as an alternative to the (-)-DIP-Cl-mediated asymmetric reduction of a key intermediate of montelukast. Org Process Res Dev. 2010;14:193-8.

54. Navari RM. Aprepitant: a neurokinin-1 receptor antagonist for the treatment of chemotherapy-induced nausea and vomiting. Expert Rev Anticancer Ther. 2004:4:715-24.

55. Naud F, Malan C, Spindler F, Rüggeberg C, Schmidt AT, Blaser H-U. Ru-(phosphine-oxazoline) complexes as effective, industrially viable catalysts for the enantioselective hydrogenation of aryl ketones. Adv Synth Catal. 2006;348:47-50.

56. Forde PM, Rudin CM. Crizotinib in the treatment of non-small-cell lung cancer. Expert Opin Pharmacother. 2012;13:1195-201.

57. Hashiguchi S, Fujii A, Takehara J, Ikariya T, Noyori R. Asymmetric transfer hydrogenation of aromatic ketones catalyzed by chiral ruthenium(II) complexes. J Am Chem Soc. 1995;117:7562-3.

58. Tanaka K, Katsurada M, Ohno F, Shiga Y, Oda M, Miyagi M, et al. Practical asymmetric synthesis of (S)-MA20565, a wide-spectrum agricultural fungicide. J Org Chem. 2000;65:432-7.
59. Lipshutz BH, Noson K, Chrisman W. Ligand-accelerated, coppercatalyzed asymmetric hydrosilylations of aryl ketones. J Am Chem Soc. 2001;123:12917-8.

60. Luesch H, Moore RE, Paul VJ, Mooberry SL, Corbett TH. Isolation of dolastatin 10 from the marine cyanobacterium Symploca species VP642 and total stereochemistry and biological evaluation of its analogue symplostatin 1. J Nat Prod. 2001;64:907-10.

61. Yasbin RE, Matthews CR, Clarke MJ. Mutagenic and toxic effects of ruthenium. Chem Biol Interact. 1980;31:355-65.

62. Cue BW, Zhang J. Green process chemistry in the pharmaceutical industry. Green Chem Lett Rev. 2009;2:193-211.

63. Farlow M, Anand R, Messina J, Hartman R, Veach J. A 52-week study of the efficacy of rivastigmine in patients with mild to moderately severe Alzheimer's disease. Eur Neurol. 2000;44:236-41.

64. Herman GA, Bergman A, Liu F, Stevens C, Wang AQ, Zeng W, et al. Pharmacokinetics and pharmacodynamic effects of the oral DPP-4 inhibitor sitagliptin in middle-aged obese subjects. J Clin Pharmacol. 2006;46:876-86.

65. Zhao H, van der Donk WA. Regeneration of cofactors for use in biocatalysis. Curr Opin Biotechnol. 2003;14:583-9.

66. Studier FW. Use of bacteriophage T7 lysozyme to improve an inducible T7 expression system. J Mol Biol. 1991;219:37-44

67. Biasini M, Bienert S, Waterhouse A, Arnold K, Studer G, Schmidt T, et al. SWISS-MODEL: modelling protein tertiary and quaternary structure using evolutionary information. Nucleic Acids Res. 2014;42:W252-8.

68. Laskowski RA, Moss DS, Thornton JM. Main-chain bond lengths and bond angles in protein structures. J Mol Biol. 1993;231:1049-67.

69. Laskowski RA, Watson JD, Thornton JM. ProFunc: a server for predicting protein function from 3D structure. Nucleic Acids Res. 2005;33:W89-93.

70. Chen I-J, Foloppe N. Drug-like bioactive structures and conformational coverage with the LigPrep/ConfGen suite: comparison to programs MOE and catalyst. J Chem Inf Model. 2010;50:822-39.

71. Mohamadi F, Richards NGJ, Guida WC, Liskamp R, Lipton M, Caufield C, et al. Macromodel; an integrated software system for modeling organic and bioorganic molecules using molecular mechanics. J Comput Chem. 1990;11:440-67.

72. Friesner RA, Banks JL, Murphy RB, Halgren TA, Klicic JJ, Mainz DT, et al. Glide: a new approach for rapid, accurate docking and scoring. 1. Method and assessment of docking accuracy. J Med Chem. 2004;47:1739-49.

73. Jakalian A, Jack DB, Fast Bayly Cl. efficient generation of high-quality atomic charges. AM1-BCC model: II. Parameterization and validation. J Comput Chem. 2002;23:1623-41.

74. Wang J, Wang W, Kollman PA, Case DA. Automatic atom type and bond type perception in molecular mechanical calculations. J Mol Graph Model. 2006;25:247-60
Ready to submit your research? Choose BMC and benefit from:

- fast, convenient online submission

- thorough peer review by experienced researchers in your field

- rapid publication on acceptance

- support for research data, including large and complex data types

- gold Open Access which fosters wider collaboration and increased citations

- maximum visibility for your research: over $100 \mathrm{M}$ website views per year

At BMC, research is always in progress.

Learn more biomedcentral.com/submissions 